\title{
Human milk oligosaccharides reduce Entamoeba histolytica attachment and cytotoxicity in vitro
}

\author{
Evelyn Jantscher-Krenn ${ }^{1} \dagger$, Tineke Lauwaet ${ }^{2}$, Laura A. Bliss $^{1}$, Sharon L. Reed ${ }^{2}$, Frances D. Gillin ${ }^{2}$ \\ and Lars Bode ${ }^{1 *}$ \\ ${ }^{1}$ Division of Neonatal Medicine and Division of Pediatric Gastroenterology and Nutrition, Department of Pediatrics, \\ University of California, 200 West Arbor Drive, MC 8450, San Diego, CA 92103-8450, USA \\ ${ }^{2}$ Department of Pathology, University of California, San Diego, CA, USA
}

(Submitted 5 October 2011 - Final revision received 7 December 2011 - Accepted 9 December 2011 - First published online 23 January 2012)

\section{Abstract}

Human milk oligosaccharides (HMO), complex sugars that are highly abundant in breast milk, block viral and bacterial attachment to the infant's intestinal epithelium and lower the risk of infections. We hypothesised that HMO also prevent infections with the protozoan parasite Entamoeba histolytica, as its major virulence factor is a lectin that facilitates parasite attachment and cytotoxicity and binds galactose (Gal) and $N$-acetyl-galactosamine. HMO contain Gal, are only minimally digested in the small intestine and reach the colon, the site of $E$. histolytica infection. The objective of the present study was to investigate whether HMO reduce E. histolytica attachment and cytotoxicity. Our in vitro results show that physiological concentrations of isolated, pooled HMO detach E. histolytica by more than $80 \%$. In addition, HMO rescue E. histolytica-induced destruction of human intestinal epithelial HT-29 cells in a dose-dependent manner. The cytoprotective effects were structure-specific. Lacto- $N$-tetraose with its terminal Gal rescued up to $80 \%$ of the HT-29 cells, while HMO with fucose $\alpha 1-2$-linked to the terminal Gal had no effect. Galacto-oligosaccharides (GOS), which also contain terminal Gal and are currently added to infant formula to mimic some of the beneficial effects of HMO, completely abolished E. histolytica attachment and cytotoxicity at $8 \mathrm{mg} / \mathrm{ml}$. Although our results need to be confirmed in vivo, they may provide one explanation for why breast-fed infants are at lower risk of E. histolytica infections. HMO and GOS are heat tolerant, stable, safe and in the case of GOS, inexpensive, which could make them valuable candidates as alternative preventive and therapeutic anti-amoebic agents.

Key words: Breast milk: Oligosaccharides: Amoebiasis

Amoebiasis, caused by the protozoan Entamoeba histolytica, is the third leading cause of death by parasitic diseases, surpassed only by malaria and schistosomiasis ${ }^{(1)}$. Worldwide, approximately 50 million people are infected with E. histolytica, resulting in nearly 100000 deaths annually. E. histolytica is transmitted by the faecal-oral route through contaminated food or water and is often endemic in regions with poor hygiene. Infection is initiated through ingestion of the semidormant cyst form of the parasite, which passes through the stomach and small intestine to reach the colon, where it differentiates into motile trophozoites that can colonise and invade the host's mucosa and cause dysentery. In rare cases, trophozoites metastasise from the colon and cause extra-intestinal abscesses mainly in the liver, and also in the lung or the brain $^{(1)}$. Colonisation and invasion require the attachment of trophozoites to the host's mucosa. Trophozoites that cannot attach are carried downstream and are excreted with the faeces without causing disease.

Breast-fed infants are at lower risk of acquiring $E$. histolytica infections than formula-fed infants ${ }^{(2)}$. This could be explained by a reduced exposure to contaminated water that might be used to prepare formula and/or by amoebicidal components in human milk. The latter has been supported by studies showing that human milk kills trophozoites ${ }^{(2,3)}$. While most studies focused on the amoebicidal activity of milk proteins such as soluble IgA, lactoferrin and lysozyme ${ }^{(4)}, 1$ litre of human milk also contains more than 10-15g of unbound complex oligosaccharides (reviewed in Newburg et al. ${ }^{(5)}$, Kunz et al. ${ }^{(6)}$ and Bode ${ }^{(7,8)}$ ),

Abbreviations: 2'FL, 2'-fucosyllactose; DP, degree of polymerisation; FPLC, fast protein liquid chromatography; Fuc, fucose; Gal, galactose; GalNAc, $N$ acetylgalactosamine; Glc, glucose; GlcNAc, $N$-acetylglucosamine; GOS, galacto-oligosaccharides; HMO, human milk oligosaccharides; HPLC-FL, HPLC with fluorescence detection; LNFP1, lacto- $N$-fucopentaose 1 ; LNFP2, lacto- $N$-fucopentaose 2 ; LNFP3, lacto- $N$-fucopentaose; LNFPx, the mixture of lacto$N$-fucopentaose 1 , lacto- $N$-fucopentaose 2 and lacto- $N$-fucopentaose 3; LNT, lacto- $N$-tetraose.

*Corresponding author: Dr L. Bode, fax +1 619543 7537, email lbode@ucsd.edu

†E. J. K. and T. L. made equal contributions. 
which exceed the amount of total milk proteins. In addition to their prebiotic effects, human milk oligosaccharides (HMO) have been reported to protect the breast-fed infant from infection with viral and bacterial pathogens such as Campylobacter jejuni, pathogenic Escherichia coli strains, Vibrio cholerae, as well as certain Shigella and Salmonella strains (reviewed in Newburg et $\left.a l{ }^{(5)}\right)$. To establish infection, these pathogens bind with their surface lectins to glycans on the luminal side of the host's intestinal epithelial cells. HMO serve as decoy glycan receptors, block the pathogens from binding to the host's epithelium and prevent infection ${ }^{(5,9)}$. E. bistolytica also employs a lectin that is involved in parasite attachment and host cell toxicity ${ }^{(10,11)}$. Whether HMO affect $E$. histolytica attachment and cytotoxicity, has not been described.

The E. bistolytica lectin recognises galactose (Gal), $N$-acetylgalactosamine (GalNAc) and lactose ${ }^{(12)}$, and HMO contain some of these sugars. HMO consist of five monosaccharide building blocks: glucose (Glc), $N$-acetylglucosamine (GlcNAc), Gal, fucose (Fuc) and sialic acid. Lactose forms the reducing end and can be elongated by one or more lacto- $N$-biose or lactosamine disaccharides (Gal $\beta 1-3 / 4 \mathrm{GlcNAc}$ ) to yield complex oligosaccharides. Lactose or the (poly-)lacto- $N$-biose/ lactosamine backbone is often fucosylated and/or sialylated to create the variety of more than 150 different $\mathrm{HMO}$ identified to date (reviewed in Newburg et al. ${ }^{(5)}$, Kunz et al. ${ }^{(6)}$ and Bode ${ }^{(7,8)}$ ).

Bovine milk-based infant formulas contain only trace amounts of HMO. In an attempt to mimic some of the health benefits of $\mathrm{HMO}$, formula manufacturers have begun to fortify their products with non-digestible galacto-oligosaccharides (GOS). GOS are synthesised enzymatically and are built out of 1-7 Gal residues attached to a Glc molecule at the reducing end. GOS have been reported to be prebiotic and to promote an intestinal microbiota composition which resembles that of breast-fed infants ${ }^{(13,14)}$. Recently, GOS have been shown to competitively inhibit $V$. cholerae toxin binding to its monosialotetrahexosylganglioside (GM1) receptor on the host cell surface $^{(15)}$

Although in vitro studies have shown that Gal, GalNAc and lactose inhibit trophozoite attachment and host cell cytotoxicity $^{(12,16)}$, these mono- and disaccharides are mostly digested and absorbed in the small intestine and are usually not found in the colon, the site of E. histolytica colonisation. In contrast, HMO and GOS resist digestion and reach the colon ${ }^{(17-20)}$. Based on their structural composition and their occurrence in the colon, we hypothesise that HMO and/or GOS interact with $E$. histolytica attachment and cytotoxicity. The present study aimed to examine the effects of isolated HMO and GOS on E. bistolytica trophozoite viability, attachment and cytotoxicity in in vitro co-cultures with human enterocytes.

\section{Materials and methods}

\section{Human milk oligosaccharide standards}

For the present study, $2^{\prime}$-fucosyllactose ( $\left.2^{\prime} \mathrm{FL}\right)$, 3-fucosyllactose, $3^{\prime}$-sialyllactose, $\sigma^{\prime}$-sialyllactose, lacto- $N$-tetraose (LNT) and lacto$N$-fucopentaose 1 (LNFP1) were purchased from V-Laboratories. LNFPx, the mixture of LNFP1, lacto- $N$-fucopentaose 2 (LNFP2) and lacto- $N$-fucopentaose 3 (LNFP3), was generously provided by Dr James Paulson (The Scripps Research Institute, La Jolla, CA, USA). GOS syrup (Vivinal, DM 75\%) was generously provided by Friesland Campina Domo. Apart from 59\% GOS, the product also contains $21 \%$ lactose, $19 \%$ Glc and $1 \%$ Gal.

\section{Isolation of pooled human milk oligosaccharides from human milk}

Human milk was obtained from eight healthy volunteers recruited at the UCSD Medical Center (San Diego, CA, USA) and approved by the University's Institute Review Board. Oligosaccharides were isolated from pooled human milk as previously described ${ }^{(21)}$. Briefly, after centrifugation, the lipid layer was removed and proteins were precipitated from the aqueous phase by the addition of ice-cold ethanol and subsequent centrifugation. Ethanol was removed from the HMO-containing supernatant by roto-evaporation. Lactose and salts were removed by size-exclusion chromatography.

\section{Cell culture}

Trophozoites of the E. histolytica strain HM-1:IMSS (isolated from a human subject in Mexico, obtained from the Instituto Mexicano del Seguro Social, Mexico City) were grown axenically in $9 \mathrm{ml}$ glass screw cap tubes in $8 \mathrm{ml}$ TYI-S-33 medium at $37^{\circ} \mathrm{C}^{(22)}$. Logphase trophozoites were harvested by chilling at $4^{\circ} \mathrm{C}$, concentrated by centrifugation $(300 \mathrm{~g}, 5 \mathrm{~min}$ ) and resuspended in the appropriate medium at the specified concentration (see next).

The human colon adenocarcinoma HT-29 cell line was cultured in Dulbecco's modified Eagle's medium supplemented with $10 \%$ heat-inactivated fetal calf serum, $60.6 \mu \mathrm{g} / \mathrm{ml}$ penicillin and $100 \mu \mathrm{g} / \mathrm{ml}$ streptomycin in a $5 \% \mathrm{CO}_{2}$ incubator at $37^{\circ} \mathrm{C}$. Following this, $7.5 \times 10^{4}$ cells were seeded in wells of a ninety-six-well plate and used after reaching confluency $(5 \mathrm{~d})$.

\section{Attachment assay}

E. histolytica trophozoites were resuspended at a concentration of $1 \times 10^{5}$ cells $/ \mathrm{ml}$ TYI-S-33 and allowed to attach to the glass of a $2 \mathrm{ml}$ vial for $1 \mathrm{~h}$ at $37^{\circ} \mathrm{C}$. Subsequently, the medium was replaced and attached trophozoites were incubated with different concentrations of $\mathrm{HMO}$ or GOS in TYI-S-33 for specified times at $37^{\circ} \mathrm{C}$. At the end of the incubation, tubes were inverted three times and detached trophozoites were collected and kept on ice. The remaining attached trophozoites were detached in ice-cold PBS for 5-10 min. Detached and 'attached' trophozoites were separately concentrated by centrifugation $\left(300 \mathrm{~g}, 5 \mathrm{~min}, 4^{\circ} \mathrm{C}\right)$ and counted in a Bürker chamber. The sum of attached and detached trophozoites was considered $100 \%$. To assess reattachment, detached trophozoites were washed and resuspended in fresh TYI-S-33 and allowed to reattach for $2 \mathrm{~h}$ at $37^{\circ} \mathrm{C}$.

\section{Cytotoxicity assay}

E. histolytica trophozoites were resuspended at a concentration of $5 \times 10^{5} \mathrm{cells} / \mathrm{ml}$ in $100 \mu \mathrm{l} /$ well HT-29 growth medium in the 
presence or absence of HMO or GOS, seeded onto HT-29 cell monolayers and incubated at $37^{\circ} \mathrm{C}$ for the time indicated. Control cell layers were incubated alone or in the presence of HMO or GOS. Co-cultures were stopped after $2 \mathrm{~h}$, and the majority of trophozoites were removed by four washes with ice-cold PBS. Co-cultures were fixed with $4 \%$ paraformaldehyde for $10 \mathrm{~min}$ at $37^{\circ} \mathrm{C}$. Destruction of HT-29 cell layers was measured as described previously ${ }^{(23)}$. Briefly, fixed cell layers were stained with $0 \cdot 1 \%$ methylene blue for $10 \mathrm{~min}$ and washed three times with PBS. The cell-incorporated methylene blue was extracted with $0 \cdot 1 \mathrm{M}-\mathrm{HCl}$ and measured in a spectrophotometer at $660 \mathrm{~nm}$. The extent of HT-29 cell layer destruction is inversely related to the optical density at $660 \mathrm{~nm}$. Because of the day-today variability in trophozoite cytotoxicity, we expressed the cytoprotective effect of HMO as '\% cell rescue' where untreated co-cultures are considered $0 \%$.

\section{Entamoeba viability assay}

Trophozoites were allowed to attach to the wall of $2 \mathrm{ml}$ glass vials for $1 \mathrm{~h}$ at $37^{\circ} \mathrm{C}$. Subsequently, the media was replaced and attached trophozoites were incubated with or without $20 \mathrm{mg} / \mathrm{ml} \mathrm{HMO}$ or $8 \mathrm{mg} / \mathrm{ml}$ GOS for $2 \mathrm{~h}$. Cell viability was determined by Trypan blue exclusion.

\section{Separation and identification of human milk}

oligosaccharides and galacto-oligosaccharides by HPLC with fluorescence detection and MS analysis

HMO and GOS were fluorescently labelled with 2-aminobenzamide and separated by HPLC on an amide- 80 column ( $4.6 \mathrm{~mm}$ inner diameter $\times 25 \mathrm{~cm}, 5 \mu \mathrm{m}$; Tosoh Bioscience) with a $50 \mathrm{~mm}$-ammonium formate-acetonitrile buffer system (L Bode et al., unpublished results). Separation was performed at $25^{\circ} \mathrm{C}$ and monitored by a fluorescence detector at $360 \mathrm{~nm}$ excitation and $425 \mathrm{~nm}$ emission.

Peak-fraction samples were lyophilised and taken up in $50 \%$ methanol-water and analysed by MS on a Thermo LCQ Duo Ion trap mass spectrometer equipped with a Nano-ESI-source. MS was carried out in positive-ion mode. Sodiated ions were detected with $\mathrm{m} / z$ values increased by $120 \mathrm{Da}$ corresponding to the 2-aminobenzamide label. All mass spectra were acquired over approximately $30 \mathrm{~s}$ in a mass/charge range of 300-2000 Da.

\section{Galacto-oligosaccharide fractionation by fast protein liquid chromatography}

GOS were subjected to automated size-exclusion fast protein liquid chromatography (FPLC) (BioLogic Duo Flow; Bio-Rad) on a P-2 biogel column (Bio-Rad). Fractions were collected in $1 \mathrm{ml}$ aliquots and their glycan content monitored by spotting $1 \mu \mathrm{l}$ aliquots onto a silica plate and staining with $5 \% \mathrm{H}_{2} \mathrm{SO}_{4}$ in methanol. The forty-two glycan-positive fractions were pooled in threes and the fourteen resulting fractions were adjusted to $8 \mathrm{mg} / \mathrm{ml}$ total GOS. Oligosaccharide composition in each fraction was determined by HPLC with fluorescence detection (HPLC-FL).

\section{Enzymatic digest}

Following these, $2^{\prime} \mathrm{FL}(1.65 \mathrm{mg}, 3.38 \mu \mathrm{mol})$ was incubated with or without $10 \mu \mathrm{l} \alpha 1,2$-fucosidase (New England BioLabs) in $100 \mu \mathrm{l}$ reaction buffer for $84 \mathrm{~h}$ at $37^{\circ} \mathrm{C}$. Reactions were stopped by enzyme inactivation at $65^{\circ} \mathrm{C}$ for $20 \mathrm{~min}$. Complete digest of $2^{\prime} \mathrm{FL}$ was confirmed by HPLC-FL analysis.

\section{Statistical analysis}

Results are expressed as mean values and standard deviations of three independent sets of experiments. Each condition was performed in duplicate for the detachment assays and in three to five replicates for the cytotoxicity assays. Data sets were analysed using one-way ANOVA followed by Dunnett's multiple comparison test. HMO-mediated detachment of E. histolytica trophozoites over time and differences in the cytotoxicity assays in the presence of GOS or the corresponding lactose concentrations were analysed using two-way ANOVA followed by Bonferroni multiple comparison test. To test for differences between individual HMO in the cytotoxicity assay, one-way ANOVA was followed by Bonferroni post-test. The level of significance was set at $P<0.05$ (Graph Pad Prism 5.0; GraphPad Software) and indicated with one asterisk; two asterisks indicate high significance $(P<0 \cdot 01)$ and three asterisks indicate extreme significance $(P<0 \cdot 001)$.

\section{Results}

\section{Human milk oligosaccharides detach Entamoeba histolytica trophozoites}

Since lactose has been reported to affect E. histolytica attachment and cytotoxicity ${ }^{(12)}$, we first analysed the lactose content of the isolated, pooled HMO by HPLC-FL and found it to comprise less than $2 \%$ of all glycans in our preparation (Fig. 1). We then incubated attached E. histolytica trophozoites with the isolated, pooled $\mathrm{HMO}$ at the specified concentrations and times and found that HMO detached E. histolytica trophozoites in a dose-dependent manner (Fig. 2(a)). At $10 \mathrm{mg} / \mathrm{ml}$, the average HMO concentration in mature human milk, HMO detached more than $80 \%$ of the trophozoites within the first $30 \mathrm{~min}$ of exposure. Light microscopic observations showed that detached trophozoites rounded up and floated in clusters (Fig. 2(b)). Since trophozoites have been reported to be susceptible to osmolality changes ${ }^{(24,25)}$, we verified that HMO did not alter the osmolality of the media (data not shown). In fact, Trypan blue staining revealed that HMO did not kill the trophozoites. After incubation with HMO $(10 \mathrm{mg} / \mathrm{ml})$ for $2 \mathrm{~h}, 98 \cdot 1$ (sD 2.0$) \%$ of the trophozoites remained viable, which was similar to the untreated controls (93.8 (SD 2.6)\%). The majority of trophozoites re-attached after the removal of HMO, which confirmed that HMO do not kill the trophozoites (data not shown). We determined the amount and composition of HMO in the culture media after $6 \mathrm{~h}$ of incubation with trophozoites and found no significant HMO loss or degradation. This indicates that trophozoites do not metabolise HMO (data not shown). 

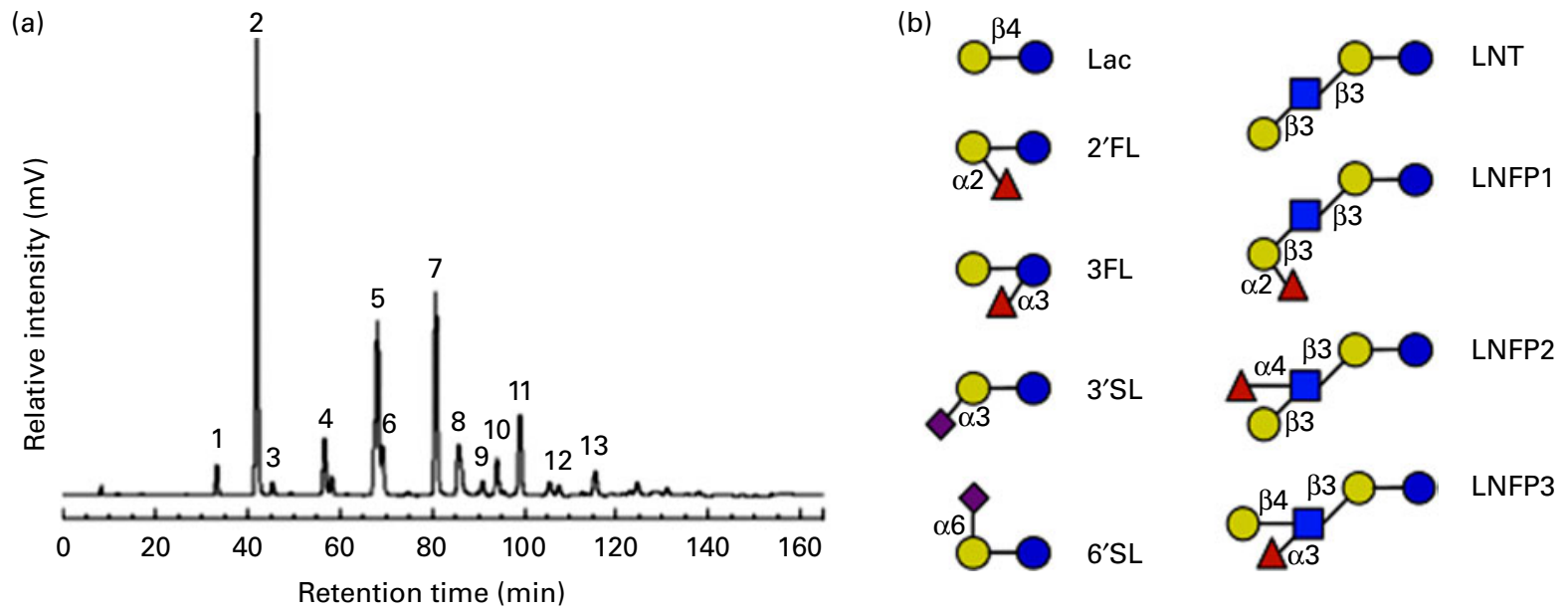

Fig. 1. HPLC with fluorescence detection (HPLC-FL) spectrum of isolated, pooled human milk oligosaccharides (HMO). (a) Representative HPLC-FL chromatogram of pooled HMO. The numbered peaks represent the following oligosaccharides: 1: lactose (Lac); 2: 2'-fucosyllactose (2'FL); 3: 3-fucosyllactose (3FL); 4: $3^{\prime}$-sialyllactose ( $3^{\prime} \mathrm{SL}$ ); 5: lacto- $N$-tetraose (LNT); 6: lacto- $N$-neo-tetraose; 7: lacto- $N$-fucopentaose 1 (LNFP1); 8: lacto- $N$-fucopentaose 2 (LNFP2); 9 : sialyl-lacto- $N$-tetraose b; 10: sialyl-lacto- $N$-tetraose c; 11 : lacto- $N$-difuco-hexaose; 12 : disialyl-lacto- $N$-tetraose; 13 : fucosyl-lacto- $N$-hexaose. (b) Structural comparison of individual HMO. Symbols represent the following monosaccharides: blue circle: glucose; yellow circle: galactose; blue square: $\mathrm{N}$-acetyl-glucosamine; red triangle: fucose; purple diamond: $N$-acetyl-neuraminic acid. $6^{\prime} \mathrm{SL}, 6^{\prime}$-sialyllactose; LNFP3, lacto- $N$-fucopentaose 3 . (A colour version of this figure can be found online at http://www.journals.cambridge.org/bjn)

\section{Human milk oligosaccharides reduce Entamoeba histolytica-induced destruction of intestinal epithelial cells}

E. histolytica trophozoites kill and phagocytose human intestinal epithelial cells in vivo and in co-culture ${ }^{(26)}$. We asked whether HMO can prevent the destruction of intestinal epithelial cells. E. histolytica trophozoites destroyed HT-29 monolayers within $2 \mathrm{~h}$ (Fig. 3(a)). Adding HMO at the beginning of the co-cultures rescued most of the HT-29 monolayers from $E$. histolytica-induced cytotoxicity (Fig. 3(a)). The effects were dose-dependent within a concentration range typical of human milk (Fig. 3(b)). To compare the effects of HMO with the previously described effects of other glycans, we estimated the average molecular weight of $\mathrm{HMO}$ as $1000 \mathrm{~g} / \mathrm{mol}$. Thus, a $10 \mathrm{mg} / \mathrm{ml} \mathrm{HMO}$ concentration would equal $10 \mathrm{~mm}$. Based on this assumption, we found that HMO were as effective in preventing $E$. histolytica-induced cytotoxicity as lactose and much more effective than Gal (Fig. 3(c)). Glc and Fuc alone did not prevent cytotoxicity, which confirms previous reports ${ }^{(12)}$.

To assess whether HMO can rescue intestinal epithelial cells that had already been in contact with E. histolytica trophozoites, we initiated co-cultures of HT-29 cells and E. histolytica trophozoites in the absence of HMO and then added HMO to the co-cultures after varying periods of time. We found that 31.0 (SD 4.7 ) \% of the intestinal epithelial cells could still be rescued when HMO were added to the co-cultures as late as $90 \mathrm{~min}$ after co-culture initiation (Fig. 4(a)). These results suggest that HMO can prevent both initiation and progression of E. histolytica-induced host cell cytotoxicity.

\section{The protective effects of human milk oligosaccharides are structure-specific}

HMO are complex mixtures of structurally diverse oligosaccharides (reviewed in Newburg et al. ${ }^{(5)}$, Kunz et al. ${ }^{(6)}$ and Bode $^{(8)}$ ). To assess which of the individual HMO are effective in the cytotoxicity assay, we tested six commercially available HMO in physiologically relevant concentrations. While lactose is a potent inhibitor of E. histolytica-induced cytotoxicity, it is most remarkable that the fucosylated and sialylated forms of lactose failed to prevent intestinal epithelial cell destruction (data not shown). Of the individual HMO tested, only LNT, a neutral unsubstituted tetrasaccharide (see Fig. 1(b)), significantly protected the intestinal epithelial cells (Fig. 4(b)). In contrast, LNFP1, a fucosylated form of LNT had no effect on rescuing the intestinal epithelial cells. Since the only structural difference between LNT and LNFP 1 is a Fuc residue $\alpha 1-2$ linked to the terminal Gal (see Fig. 1(b)), our findings suggest that the terminal Gal on HMO is required for their cytoprotective effect. To confirm this hypothesis, we tested equimolar concentrations of LNT and LNFP1 in the cytotoxicity assay. LNT had a dose-dependent effect reaching 79.0 (SD 8.3) \% cell rescue at $10 \mathrm{~mm}$, whereas the effect of an equimolar concentration of LNFP1 was significantly lower (16.9 (SD 1.7)\%) (Fig. 4(b)). Interestingly, a $10 \mathrm{~mm}$ mixture of the isomers LNFP1, LNFP2 and LNFP3 consisting of $62 \%$ LNFP1 had a significantly higher effect (61.7 (SD 5.5)\%) than 10 mm-LNFP1 alone. This suggests that LNFP2 and/or LNFP3 strongly inhibit E. histolytica-induced cytotoxicity. Both LNFP2 and LNFP3 are substituted by Fuc at the subterminal GlcNAc, leaving the terminal Gal accessible (see Fig. 1(b)). This supports our hypothesis that the terminal $\mathrm{Gal}$ is required for the cytoprotective effect of HMO. To further verify this hypothesis, we used 2'FL (see Fig. 1(b)), which also carries an $\alpha 1-2$-linked Fuc residue at the terminal Gal, and removed the Fuc with a specific $\alpha 1-2$-fucosidase as confirmed by HPLC-FL and MS (data not shown). While the intact $2^{\prime} \mathrm{FL}$ had no cytoprotective effect, enzymatic removal of Fuc from the terminal Gal resulted in a significant cytoprotective effect similar to that of equimolar concentrations of either lactose alone or a mixture of lactose and Fuc (Fig. 4(c)). These results strongly 


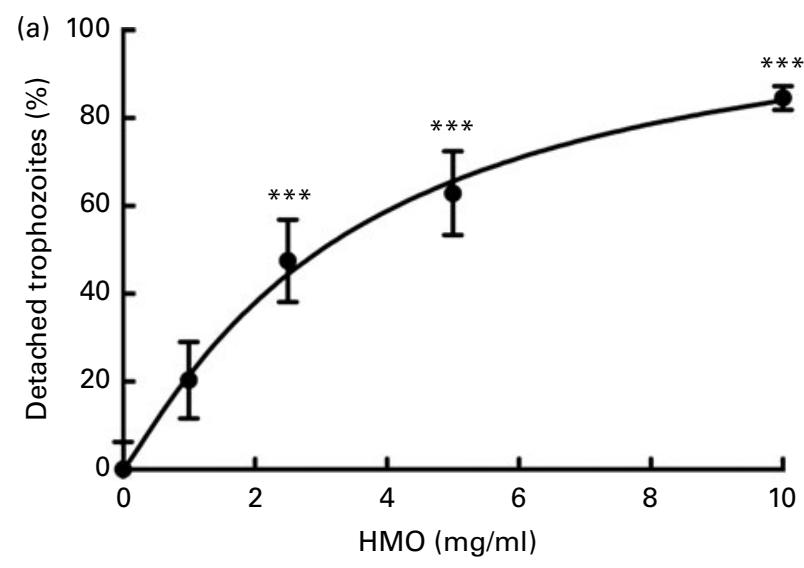

(b)

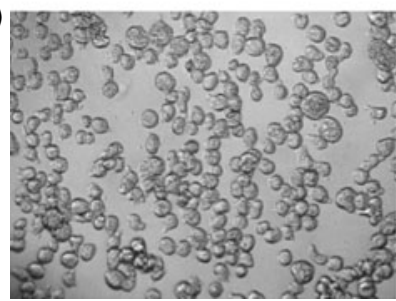

Eh

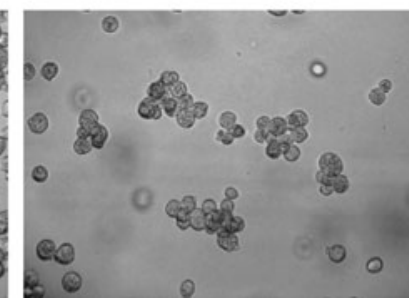

$\mathrm{Eh}+\mathrm{HMO}(10 \mathrm{mg} / \mathrm{ml})$
Fig. 2. Human milk oligosaccharides (HMO) detach Entamoeba histolytica trophozoites. (a) Attached E. histolytica trophozoites were incubated with isolated, pooled $\mathrm{HMO}$ at specified concentrations for $30 \mathrm{~min}$. Detached and attached cells were counted and the percentage of detached cells was calculated and normalised to HMO-untreated controls. Data points represent the means and standard deviations of three independent experiments performed in duplicate. ${ }^{* * *}$ Mean values were significantly different $(P<0.001)$. (b) Representative light microscopic images of control (left) and HMO-treated (right) E. histolytica trophozoites (Eh).

suggest that the blockage of the terminal Gal, not the presence of Fuc per se, renders $2^{\prime} \mathrm{FL}$ ineffective.

\section{Galacto-oligosaccharides prevent Entamoeba histolytica-} induced cytotoxicity independent of its lactose content

GOS, which are widely added to infant formula as a prebiotic, consist of varying numbers of $G$ al residues attached to a single Glc at the reducing end. Since our data indicate that terminal $\mathrm{Gal}$ is essential for the cytoprotective effect of HMO, we tested whether GOS are also effective against $E$. histolytica-induced cytotoxicity. We found that GOS indeed protect HT-29 cell monolayers from destruction by trophozoites in a dosedependent manner (Fig. 5(a)). At only $5 \mathrm{mg} / \mathrm{ml}$, GOS fully protected HT-29 cells from $E$. histolytica-induced cytotoxicity.

GOS are synthesised enzymatically by the addition of Gal to lactose. Hence, the resulting mixture of oligosaccharides also contains unreacted lactose and monosaccharides. To rule out that the cytoprotective effect is due to the unreacted lactose only, we tested lactose at the respective concentrations present in the GOS preparation and found that the effects of lactose alone were significantly lower than the effects of the lactose-containing GOS preparation (Fig. 5(a)). These results suggest that the GOS preparation contains oligosaccharides other than lactose that prevent cytotoxicity. To confirm these results, we separated the oligosaccharides in the GOS preparation by size-exclusion chromatography and determined the lactose content and cytoprotective effect of the resulting fourteen fractions. Consistent with their high lactose content, fractions 13 and 14 have a high cytoprotective effect on co-cultures (Fig. 5(b)). HPLC-FL analysis of the different FPLC fractions followed by MS analysis of the HPLC peaks showed that FPLC fractions 10-12 contain almost exclusively trisaccharides (degree of polymerisation (DP)3) and hardly any lactose. These fractions had significant cytoprotective effects. Fractions 6-9 contain predominantly tetrasaccharides (DP4) and mediate lower but still significant cell rescue. Early eluted fractions containing larger oligosaccharides (DP4-DP8) had no effect on cytotoxicity, which might be due to the much lower abundance of oligosaccharides DP $>4$ in the GOS preparation (Fig. 5(c)). We prepared lactose 'free' GOS ( $<0.5 \%$ lactose) by pooling all GOS fractions with a lactose content $\leq 3 \%$ and compared the cytoprotective effect of the pooled fractions to equal concentrations of the lactose-containing GOS preparation (Fig. 5(d)). In accordance with the additional effect of GOS compared to lactose alone (Fig. 5(a)), we found a dose-dependent cell rescue effect in lactose-free GOS, indicating a lactose-independent effect of GOS. Remarkably, at $8 \mathrm{mg} / \mathrm{ml}$, the concentration used to supplement infant formula, lactose-free GOS almost completely rescued $E$. bistolytica-induced destruction of intestinal epithelial HT-29 cells. Similar to HMO, GOS are not toxic to trophozoites (96.6 (SD 1.3)\% viable compared to 93.8 (SD 2.6)\% in the control), detach trophozoites from glass (81.3 (SD $7 \cdot 3$ ) \% detachment within $30 \mathrm{~min}$ compared to control) and provide substantial protection to cell layers in established co-cultures with E. histolytica trophozoites (Fig. 5(d)). These results confirm that the protective effect of GOS is lactoseindependent and comparable to the effects of pooled HMO.

\section{Discussion}

We have shown that physiological concentrations of HMO and formula-based concentrations of GOS inhibit $E$. bistolytica attachment and cytotoxicity to enteric cell layers in vitro in a dose-dependent manner. Interestingly, HMO and GOS also rescued cell layers from E. histolytica-induced cytotoxicity when added after the initiation of the co-cultures, indicating that HMO can halt ongoing cytotoxicity.

HMO did not affect attachment and viability of the mucosal protozoan pathogen Giardia lamblia (T Lauwaet, unpublished results), suggesting that $\mathrm{HMO}$ interfere with E. bistolyticaspecific attachment mechanisms. We speculate that HMO bind to the Gal/GalNAc lectin on the surface of E. bistolytica trophozoites. The Gal/GalNAc lectin mediates trophozoite attachment and invasion and consists of three subunits: heavy, intermediate and light. The extracellular part of the heavy subunit contains a carbohydrate recognition domain and can be inhibited by lactose, $\mathrm{Gal}$ and $\mathrm{N}$-acetyl-galactosamine but not by Glc and Fuc ${ }^{(12)}$. We found that the cytoprotective effects of HMO and GOS are not due to contaminating lactose as our isolated, pooled HMO preparation was virtually lactose free (Fig. 1(a)). Similarly, the GOS preparation still prevented $E$. histolytica attachment and cytotoxicity after lactose 
(a)

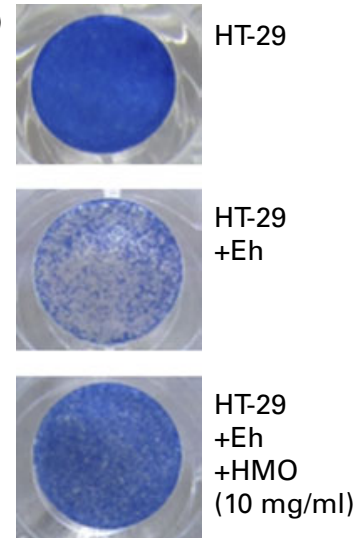

(b)

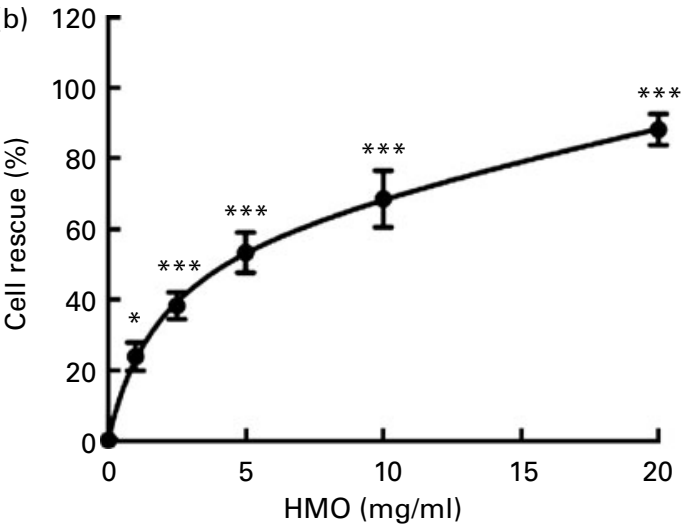

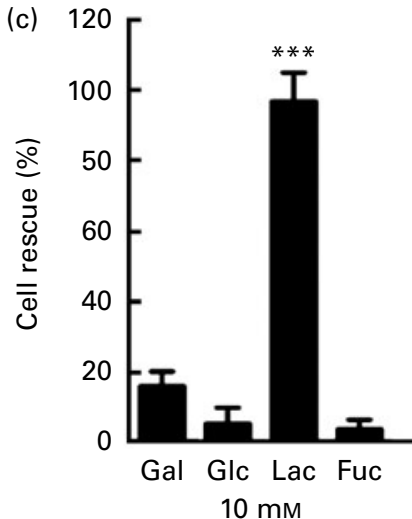

Fig. 3. Human milk oligosaccharides (HMO) prevent Entamoeba histolytica-induced destruction of intestinal epithelial cell layers. (a) Representative images of methylene blue stained HT-29 cell layers alone (top) or co-cultured with E. histolytica trophozoites (Eh) in the absence (middle) or presence of HMO (bottom). (b) HT-29 cell layers were co-cultured for $2 \mathrm{~h}$ with $E$. histolytica trophozoites in the presence or absence of HMO and cell layer destruction determined by methylene blue staining. Data points are the means and standard deviations of four independent experiments performed in duplicate and represent the percentage cell rescue. (c) Bars represent the percentage cell rescue of HT-29 cell layers co-cultured with E. histolytica trophozoites in the presence of $10 \mathrm{mM}$-galactose (Gal), glucose (Glc), lactose ( $\mathrm{Lac}$ ) or fucose (Fuc). The cytoprotective effect of lactose is comparable to that of isolated, pooled HMO in (b). Mean values were significantly different: ${ }^{\star} P<0.05,{ }^{\star * \star} P<0.001$. (A colour version of this figure can be found online at http://www.journals.cambridge.org/bjn)

removal by size-exclusion chromatography. These findings are important since most of the lactose is cleaved and absorbed in the small intestine, while HMO and GOS are only minimally digested and reach the colon ${ }^{(17,18)}$, the site of $E$. bistolytica colonisation.

Both HMO and GOS are heterogeneous mixtures of structurally diverse oligosaccharides with potentially very different effects. Here, we have shown that the cytoprotective effects of HMO are indeed structure-specific and that a free terminal Gal is necessary to prevent E. histolytica attachment and cytotoxicity. HMO are not cytoprotective if the terminal Gal is covered with an $\alpha 1-2$-linked Fuc as it is the case for $2^{\prime} \mathrm{FL}$ and LNFP1. Considering the inter-individual and genetic differences in HMO composition found in the milk of different women $^{(27,28)}$, these results may have important physiological relevance. Evidently, $2^{\prime} \mathrm{FL}$ and LNFP1 are among the most abundant oligosaccharides in the milk of so-called secretor women that express a specific $\alpha 1-2$-fucosyltransferase. In contrast, $2^{\prime}$ FL and LNFP1 are completely absent in the milk of non-secretor women who lack $\alpha 1$-2-fucosyltransferase. Based on our in vitro results, one could hypothesise that the milk of non-secretor women confers better protection against
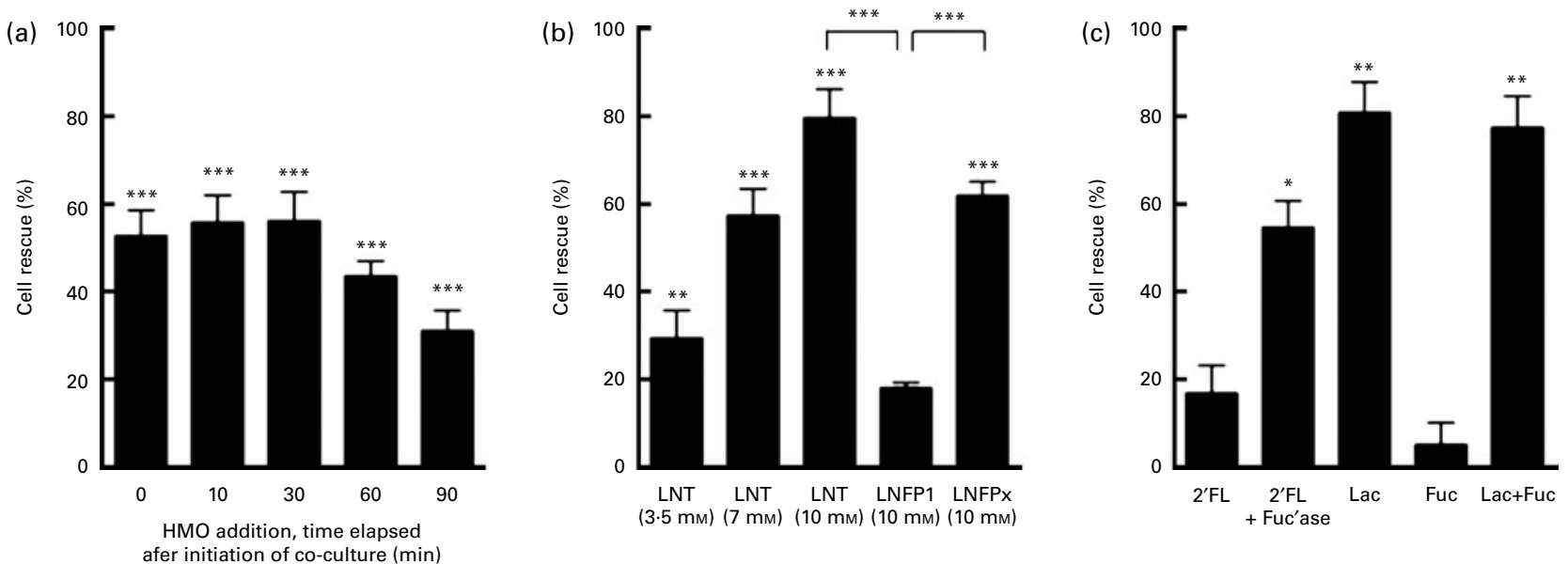

Fig. 4. Human milk oligosaccharides (HMO) rescue existing co-cultures and the effects are structure-dependent. (a) HMO rescue HT-29 intestinal epithelial cell layers when added to existing co-cultures with Entamoeba histolytica trophozoites. HT-29 cell layers were co-cultured with E. histolytica trophozoites in the absence of $\mathrm{HMO}$ for the time indicated. At that time, $\mathrm{HMO}$ were added to the co-cultures up to a total co-culture time of $2 \mathrm{~h}$. $\mathrm{HT}$-29 cells that were co-cultured with E. histolytica trophozoites in the absence of HMO for the entire $2 \mathrm{~h}$ were considered to have $0 \%$ cell rescue. (b) Lacto- $N$-tetraose (LNT) but not lacto- $N$-fucopentaose 1 (LNFP1) rescue E. histolytica-induced HT-29 cell destruction. HT-29 cells and E. histolytica trophozoites were co-cultured in the presence of individual HMO. While physiological and superphysiological concentrations of LNT rescued HT-29 cells in a dose-dependent manner, LNFP1 had no effect even at 10 mM. However, LNFPx, a mixture of LNFP1, lacto- $N$-fucopentaose 2 and lacto- $N$-fucopentaose 3 , rescued HT-29 cells. For structural differences, see Fig. 1(b). (c) Removal of terminal fucose restores cytoprotective effect. While $2^{\prime}$-fuosyllactose (2'FL) did not rescue HT-29 cells from E. histolytica-induced cytotoxicity, removal of the terminal fucose by incubation with fucosidase (Fuc'ase) restored the cytoprotective effect, which was almost as pronounced as that of lactose (Lac) alone. Fucose (Fuc) alone had no effect, but it also did not reduce the effect of lactose. Bars represent the means and standard deviations of four independent experiments. Mean values were significantly different: ${ }^{\star} P<0.05,{ }^{\star \star} P<0.01,{ }^{\star \star \star} P<0.001$. 
E. bistolytica infections than the milk of secretor women, where the majority of HMO is covered with $\alpha 1-2$-linked Fuc. The opposite has been shown for $C$. jejuni infections. C. jejuni binds to $\alpha 1-2$-fucosylated oligosaccharides ${ }^{(29)}$, and the milk of secretor women provides infants with a better protection against $C$. jejuni infections ${ }^{(30)}$. These results clearly demonstrate that some of the beneficial effects of HMO are highly structure-specific and that the amount and composition of oligosaccharides in mother's milk confer different degrees of protection against specific pathogens.

Despite vast improvements in glycan synthesis over the last few years, the still relatively high costs of chemical or enzymatic oligosaccharide synthesis are likely to limit the use of individual $\mathrm{HMO}$ as templates for anti-amoebic therapies, especially in developing countries, where amoebiasis is most prevalent. GOS, however, can be produced in large quantities and at low costs and have been used in infant formula for almost a decade now. However, the high lactose concentration in crude GOS preparations may be difficult to tolerate for amoebiasis patients who often present with secondary lactose intolerance $^{(31)}$. Instead, the use of refined, lactose-depleted GOS preparations might be an affordable anti-amoebic therapy.

Our results suggest that HMO contribute to the anti-amoebic effects of human milk, which may help explain why breast-fed infants are at lower risk to acquire E. histolytica infections ${ }^{(2)}$. Future in vivo studies are needed to confirm that regular intake of HMO and GOS can prevent and/or treat E. histolytica infections. HMO and GOS are heat tolerant, stable, safe and in the case of GOS, inexpensive. Since most areas with a high rate of $E$. bistolytica infections also have an extreme climate and very limited resources, heat tolerance, stability and low costs are valuable characteristics that make these oligosaccharides ideal candidates as alternative preventive and therapeutic anti-amoebic agents.
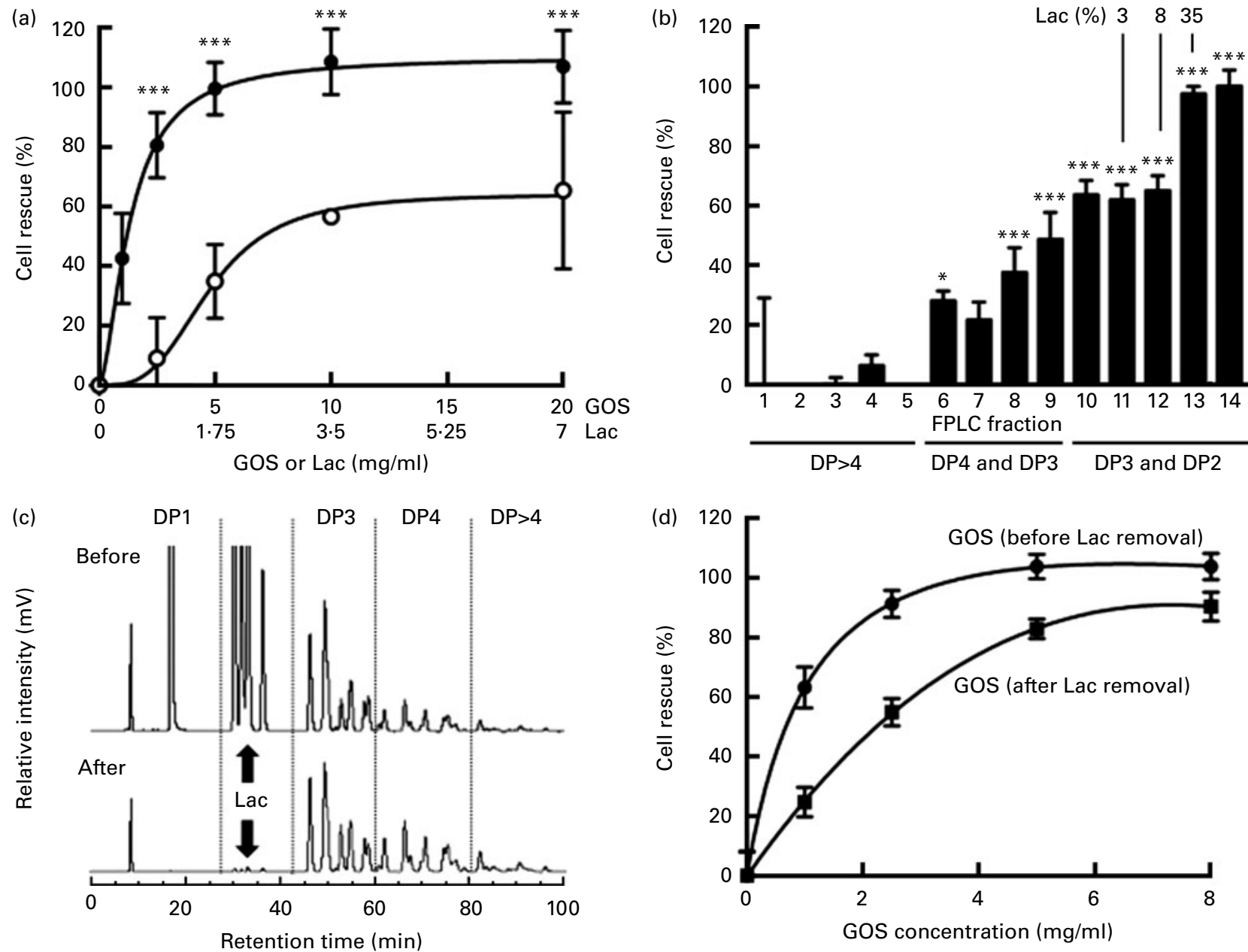

Fig. 5. Galacto-oligosaccharides (GOS) prevent Entamoeba histolytica-induced destruction of intestinal epithelial cells and the effects are lactose-independent (a) Bars show the \% cell rescue of HT-29 cell layers co-cultured with E. histolytica trophozoites and treated with GOS ( $\bullet$ ) or the concentration of lactose (Lac) (O) that is present in GOS (35\%). Data points represent the means and standard deviations of four independent experiments. ${ }^{\star \star \star}$ Mean values were significantly different between the effects of the GOS preparation and the effects of lactose alone $(P<0.001)$. (b) Cytoprotective effects of GOS size-exclusion chromatography fractions. Fast protein liquid chromatography (FPLC) size-exclusion chromatography was used to remove lactose from the GOS preparation. Early fractions contain oligosaccharides with higher molecular weights (more monosaccharide residues, higher degree of polymerisation (DP)). Later fractions contain oligosaccharides with lower molecular weight, including lactose (\% lactose is given above the bars). Bars represent the means and standard deviations of four independent experiments. (c) HPLC with fluorescence detection chromatograms of the GOS preparation before (top) and after FPLC size-exclusion chromatography (bottom). (d) Cytoprotective effects of lactose-containing GOS before FPLC size-exclusion chromatography ( $\bullet$ ) and lactose-depleted GOS ( $\mathbf{\square})$. Data points represent the means and standard deviations of four replicates. 


\section{Acknowledgements}

We are grateful to Ken Hirata for providing us with E. bistolytica culture media, to Kirin Demuynck for help with the initial attachment experiments, and Caroline Nissan and Lauren Hsiao for their technical assistance with HMO purification and analysis. We thank Dr James Paulson at The Scripps Research Institute, La Jolla, CA, for kindly providing us with LNFPx and Friesland Campina Domo for their generous gift of Vivinal GOS. This project has been funded in part by National Institutes of Health (NIH) grants K99/R00 DK078668 (L. B.), R03 HD059717S1 (L. B.) and R01 AI42488 (F. D. G.). The contributions of the authors to this study were as follows: L. B. and T. L. designed the research; E. J.-K., T. L. and L. A. B. conducted the research; L. B., E. J.-K. and T. L. analysed the data; E. J.-K., T. L., S. L. R., F. D. G. and L. B. wrote the paper. L. B. had primary responsibility for the final content. All authors read and approved the final manuscript. E. J.-K., T. L., L. A. B., S. L. R., F. D. G. and L. B. have no conflicts of interest to declare.

\section{References}

1. Pritt BS \& Clark CG (2008) Amebiasis. Mayo Clin Proc 83, 1154-1159.

2. Islam A, Stoll BJ, Ljungstrom I, et al. (1988) The prevalence of Entamoeba histolytica in lactating women and in their infants in Bangladesh. Trans $R$ Soc Trop Med Hyg 82, 99-103.

3. Gillin FD, Reiner DS \& Wang CS (1983) Human milk kills parasitic intestinal protozoa. Science 221, 1290-1292.

4. Leon-Sicairos N, Lopez-Soto F, Reyes-Lopez M, et al. (2006) Amoebicidal activity of milk, apo-lactoferrin, sIgA and lysozyme. Clin Med Res 4, 106-113.

5. Newburg DS, Ruiz-Palacios GM \& Morrow AL (2005) Human milk glycans protect infants against enteric pathogens. Annu Rev Nutr 25, 37-58.

6. Kunz C, Rudloff S, Baier W, et al. (2000) Oligosaccharides in human milk: structural, functional, and metabolic aspects. Annu Rev Nutr 20, 699-722.

7. Bode L (2009) Human milk oligosaccharides: prebiotics and beyond. Nutr Rev 67, Suppl. 2, S183-S191.

8. Bode L (2006) Recent advances on structure, metabolism, and function of human milk oligosaccharides. J Nutr $\mathbf{1 3 6}$, 2127-2130.

9. Morrow AL, Ruiz-Palacios GM, Jiang X, et al. (2005) Humanmilk glycans that inhibit pathogen binding protect breastfeeding infants against infectious diarrhea. J Nutr 135, 1304-1307.

10. Saffer LD \& Petri WA Jr (1991) Entamoeba histolytica: recognition of alpha- and beta-galactose by the $260-\mathrm{kDa}$ adherence lectin. Exp Parasitol 72, 106-108.

11. Saffer LD \& Petri WA Jr (1991) Role of the galactose lectin of Entamoeba histolytica in adherence-dependent killing of mammalian cells. Infect Immun 59, 4681-4683.

12. Cano-Mancera R \& Lopez-Revilla R (1987) Inhibition of the adhesion of Entamoeba histolytica trophozoites to human erythrocytes by carbohydrates. Parasitol Res 74, 18-22.

13. Moro G, Minoli I, Mosca M, et al. (2002) Dosage-related bifidogenic effects of galacto- and fructooligosaccharides in formula-fed term infants. I Pediatr Gastroenterol Nutr $\mathbf{3 4}$, 291-295.

14. Boehm G, Fanaro S, Jelinek J, et al. (2003) Prebiotic concept for infant nutrition. Acta Paediatr 91, 64-67.

15. Sinclair HR, de Slegte J, Gibson GR, et al. (2009) Galactooligosaccharides (GOS) inhibit Vibrio cholerae toxin binding to its GM1 receptor. J Agric Food Chem 57, 3113-3119.

16. Ravdin JI \& Guerrant RL (1981) Role of adherence in cytopathogenic mechanisms of Entamoeba bistolytica. Study with mammalian tissue culture cells and human erythrocytes. J Clin Invest 68, 1305-1313.

17. Gnoth MJ, Kunz C, Kinne-Saffran E, et al. (2000) Human milk oligosaccharides are minimally digested in vitro. J Nutr $\mathbf{1 3 0}$, 3014-3020

18. Engfer MB, Stahl B, Finke B, et al. (2000) Human milk oligosaccharides are resistant to enzymatic hydrolysis in the upper gastrointestinal tract. Am J Clin Nutr 71, 1589-1596.

19. Coppa GV, Pierani P, Zampini L, et al. (2001) Characterization of oligosaccharides in milk and feces of breast-fed infants by high-performance anion-exchange chromatography. Adv Exp Med Biol 501, 307-314.

20. Albrecht S, Schols HA, van Zoeren D, et al. (2011) Oligosaccharides in feces of breast- and formula-fed babies. Carbohydr Res 346, 2173-2181.

21. Kunz C, Rudloff S, Hintelmann A, et al. (1996) High-pH anion-exchange chromatography with pulsed amperometric detection and molar response factors of human milk oligosaccharides. J Chromatogr B Biomed Appl 685, 211-221.

22. Diamond LS, Harlow DR \& Cunnick CC (1978) A new medium for the axenic cultivation of Entamoeba histolytica and other Entamoeba. Trans R Soc Trop Med Hyg 72, 431-432.

23. Mirelman D, Bracha R \& Sargeaunt PG (1984) Entamoeba bistolytica: virulence enhancement of isoenzyme-stable parasites. Exp Parasitol 57, 172-177.

24. Gillin FD \& Diamond LS (1980) Attachment and short-term maintenance of motility and viability of Entamoeba histolytica in a defined medium. J Protozool 27, 220-225.

25. Gillin FD \& Diamond LS (1980) Attachment of Entamoeba bistolytica to glass in a defined maintenance medium: specific requirement for cysteine and ascorbic acid. J Protozool 27, 474-478.

26. Burchard GD, Prange G \& Mirelman D (1993) Interaction between trophozoites of Entamoeba bistolytica and the human intestinal cell line HT-29 in the presence or absence of leukocytes. Parasitol Res 79, 140-145.

27. Thurl S, Munzert M, Henker J, et al. (2010) Variation of human milk oligosaccharides in relation to milk groups and lactational periods. Br J Nutr 104, 1261-1271.

28. Coppa GV, Gabrielli O, Zampini L, et al. (2011) Oligosaccharides in 4 different milk groups, bifidobacteria, and Ruminococcus obeum.JPediatr Gastroenterol Nutr 53, 80-87.

29. Ruiz-Palacios GM, Cervantes LE, Ramos P, et al. (2003) Campylobacter jejuni binds intestinal $\mathrm{H}(\mathrm{O})$ antigen (Fuc alpha 1, 2Gal beta 1, 4GlcNAc), and fucosyloligosaccharides of human milk inhibit its binding and infection. $J$ Biol Chem 278, 14112-14120.

30. Morrow AL, Ruiz-Palacios GM, Altaye M, et al. (2004) Human milk oligosaccharides are associated with protection against diarrhea in breast-fed infants. J Pediatr 145, 297-303.

31. Rana SV, Bhasin DK \& Vinayak VK (2004) Prospective evaluation of lactose malabsorption by lactose hydrogen breath test in individuals infected with Entamoeba histolytica and passing cysts. Br J Nutr 92, 207-208. 